\title{
Sutured Repair of Primary Small Umbilical and Epigastric Hernia: Concomitant Rectus Diastasis is a Significant Risk-Factor for Recurrence
}

\author{
Danny Rosin
}

Published online: 18 September 2014

(C) Société Internationale de Chirurgie 2014

The use of mesh in hernia repair has become so ubiquitous that we tend to forget that "pockets of resistance" still exist, and primary suture repair is still prevalent. The two main justifications for accepting a technique with higher recurrence rate are cost and the risk of infection. The latter was dismissed by numerous studies as small and "acceptable" in most instances except for high-risk situations like incarceration with bowel perforation. With proper antibiotic prophylaxis, there is no reason to avoid a mesh in elective, clean conditions. But cost is still an issue in many places around the globe, and mesh availability is not universal.

It is imperative, tough that we know the actual outcome of non-mesh repair and decide whether it is justified. We have to remember that hernia recurrence is by itself a costly outcome. Some surgeons, like the author of the article in this issue [1], still favor a non-mesh repair, and from them, we can learn the facts. The author, Dr. Köhler, has retrospectively analyzed more than 200 of his patients who have undergone elective suture repair of "small" umbilical and epigastric hernias, defined by diameter of less than $2 \mathrm{~cm}$. His honest report of an overall recurrence rate of $18.9 \%$, over a mean follow-up of 31 months, is certainly a deterrent to adopt such a technique, but what is more interesting is the subgroup analysis which is informative and educational. Two factors were found to significantly affect the outcome-the type of suture material used and the presence of diastasis recti-each of these factors was associated with nearly $30 \%$ rate of recurrence (and together-55\%!). So, obviously, hernia repair with absorbable sutures is obsolete-a well-known fact. But the presence of rectal divarication (of more than $2 \mathrm{~cm}$ ), meticulously measured by ultrasound in all cases, is not a commonly used parameter, and its effect on the outcome is worth recognition: concentrating on non-absorbable suture repair only, the recurrence rate dropped from 16 to $5.5 \%$ in the absence of diastasis. This percentage, albeit relatively high, can be considered acceptable by some surgeons in some situations. Assessing the width of rectus muscles separation is easy-one can use a finger instead of an ultrasound machine (remember that cost is an issue here!).

So, it may be acceptable to be selective in using mesh for small midline hernias (defining "small" is a separate topic). But we also have to be selective in preferring a suture repair and take into account the multiple factors that will affect the outcome. Rectal separation is certainly an important factor that should be added to the calculation. By itself, diastasis is mainly of cosmetic importance that does not mandate repair, but it is a marker of tissue weakness, which heralds the failure of primary repair.

\section{Reference}

1. Köhler G, Luketina R-R, Emmanuel K. Sutured repair of primary small umbilical and epigastric hernias: concomitant rectus diastasis is a significant risk factor for recurrence. World J Surg. doi:10. 1007/s00268-014-2765-y
D. Rosin $(\varangle)$

The Department of General Surgery and Transplantation, Sheba

Medical Center, Ramat-Gan, Israel

e-mail: drosin@mac.com 